\title{
DIE GESCHICHTSAUFFASSUNG DES HERMENEUTISCHEN DENKENS
}

\author{
von Uirich Johannes Schneider
}

\section{Einleitung}

Jede gegenwärtig angestrengte Lektüre Schleiermachers führt unweigerlich zu jener Zeit zurück, die seit dem 19. Jahrhundert als "Aufgang der geschichtlichen Welt" (Dilthey), als Morgendämmerung des "historischen Bewußtseins" (Meinecke) gefeiert wird. Schleiermachers hermeneutische Theorie (und vorausgegangene Veröffentlichungen) scheinen am Bildungsroman der historischen und philologischen Wissenschaften zu partizipieren und die neue Evidenz des historischen Denkens auszustellen. So zeigt sich in den Reden über Religion (1799) erstmals ein Religionsbegriff, der historische Vielfalt und Verschiedenheit der religiösen Anschauungen notwendig fordert; die Monologen (1800) entwerfen die Idee von Subjektivität in der Reflexion von Welt auf "Geist"; die Hermeneutik (erste Ausarbeitungen ab 1805) ist als philosophische Kunstlehre ganz aus der geschichtlichen Erfahrung der Distanz der Interpretation zur Quelle entwickelt.

Im Programm solcher Ansätze liegt eine Philosophie der Geschichtlichkeit vorausgesetzt $t^{1}$, die Schleiermacher sowohl hermeneutisch: in der Maxime, Mißverstand überall anzunehmen, wie auch dialektisch: als Einsicht in die Sprachlichkeit des Denkens formuliert. Diese Philosophie der Geschichtlichkeit kennt, in der Theorie selbstexpressiver Subjektivität, in der Kunstlehre aneignender Auslegung des Fremden, kein Prinzip objektiv-zeitlicher Entwicklung, ist darum auch nicht Geschichtsphilosophie, nicht Rekonstruktion vorvergangener Vernunft. Anders als Hegel nimmt Schleiermacher historische Mannigfaltigkeit überhaupt nicht als Gegenstand einer Philosophie, sondern reflektiert auf sie aus dem einfachen Abstand des Jetzt zum Einst, des Eigenen zum Fremden. Daß im hermeneutischen Denken nicht Konstruktion der geschichtlichen Welt aus präsentischer Selbstgewißheit, vielmehr Nachkonstruktion aus Notwendigkeit zur Selbstkonstitution Aufgabe wird, verweist auf eine gewandelte Bestimmung von Subjektivität, für die aus Kants Transzendentalphilosophie die Einbildungskraft zum Prinzip genommen wird, das als "Phantasie" (Reden über Religion) oder, in Schellings Worten, als Vermögen der "Passivität aus Selbsttätigkeit" gilt $^{2}$.

1 S. Gunter Scholz: Ergänzungen zur Herkunft des Wortes "Geschichtlichkeit", in: Archiv f. Begriffsgesch. 14, 1970, S. $112 \mathrm{ff}$.

2 F.Schleiermacher: Über die Religion, Reden an die Gebildeten unter ihren Verăchtern (1799), Hamburg 1958, S. 70 et passim.

F.W.J.Schelling: Philsophische Briefe über Dogmatismus und Kritizismus, in: Sämt1, Werke Bd. I/1 (1856), S. 332 (Nachdruck Darmstadt 1975, S. 212). 
Indem so aus Schleiermachers Schriften radikaler als aus der idealistischen Geschichtsphilosophie der Bruch mit der aufklärerisch-rationalistischen Geschichtsauffassung gelesen werden kann, läßt sich das Pathos der spätromantischen Verklärung des historischen Bewußtseins revidieren und ineins damit begründen:

(a) Die hermeneutische Theorie hat ihre Aufgabe als philosophisch reflektierte Kunstlehre ursprünglich erst in der Bestimmung von immer schon geschichtlicher Subjektivität. Verstehen wird notwendig allgemeine Forderung, wo Vermittlung des Individuellen mit dem Allgemeinen das Gesetz der Welt ist ${ }^{3}$. Die Evidenz der Geschichtsauffassung des hermeneutischen Denkens ist so - und nicht schon allein im Regelkanon der Auslegungslehre - Bruch mit der rationalistischen, auch in $h \mathrm{l} s \mathrm{t} \circ \mathrm{r} \mathrm{i}$ a $\mathrm{k} \circ \mathrm{n} s \mathrm{t} u \mathrm{i}$ e renden Vernunft, Bruch aber als Reflexion dieser Vernunft, als Beugung ihres Anspruchs unter die Kautelen einer Dialektik der Modifikation [s.u. 2].

(b) Was philosophisch den sich historice konstituierenden Geist ausmacht, ist positiv ausweisbar an der Institution geisteswissenschaftlicher Forschung, in der sich hermeneutisches Denken verwirklicht. Daß der "denkende Verkehr mit der historischen Lebenswirklichkeit" (Troeltsch) selber Geschichte macht, provozierte seine Krise: Historismus ist der Titel für diese Reflexion hermeneutischer Praxis an ihren Ergebnissen. Dabei wird der moralische Wert der Geschichte im gleichen Maße geleugnet wie andererseits ihre quasi-natürliche Ordnung in Epochen versichert, so daß die Behauptung eines Fortschritts der Geschichte metaphysisch, die ihrer Bedeutung fast empirisch scheint (Dilthey) [s.u. 3].

(c) Jede gegenwärtige Lektüre Schleiermachers, die die antirationalistische Geschichtsauffassung des hermeneutischen Denkens revoziert, kennt - als geisteswissenschaftliche Aufgabe - auch die historische Fraktionierung der Geschichte in Epochen. Deshalb mag ihr Schleiermacher quasi gegenständlich einer Zeit zugehörig erscheinen, deren Denken gerade durch die Differenz (gleich ob der Absicht oder der Aussage nach) zum aufklärerisch-rationalistischen Denken eine a $n d e r$ Epoche macht. Diese Epoche (emphatisch 'historischen Bewußtseins') wird im historistischen Denken positiv als $u n s$ e $r$ a ausgewiesen, well sie nicht hintergangen werden kann (man bekennt sich zum Relativismus und schwört der Geschichtsphilosophie $a b)$. So wäre - in durchaus geübter, trivialer Weise - der gegenwärtigen Thematisierung von Geschichte eine Anfangsevidenz 'verortet', auf die man sich affirmativ rückbeziehen kann (- die klassischen Rehabilitierungsversuche

3 F.Schleiermacher: Hermeneutik und Kritik, hg. v. M.Frank, Frankfurt/M. 1977 , S. 80 . 
der Aufklärung in dieser Rücksicht bei Cassirer und Troeltsch waren vergebliche Einsprüche). Das gängige Geschichtsbild bannt so geistige Vergangenheit in zeitlich gegliederte Abschnitte, an denen sich die Herkunft der Gegenwart abmißt. Wie im Museum kann kontemplativ in der Vorstellung zugleich Distanz genommen und bewundert werden: Noch der einfachste Gedanke an Geschichte als etwas Positives trägt diesen Widerspruch in sich, selber nur Verhalten, nur rezeptiv sein zu wollen. Zum quasi-mythisch Unbegreiflichen wird daher die Evidenz des geschichtlichen Denkens erst in einer Einstellung des Rückblicks substantialisiert, weil zur historischen Tatsache gerade das erkoren wird, was aktual nicht (mehr) möglich scheint. Das Vergessen von Ausbildung, Entstehung erst macht nämlich das Vergangene zum toten Rest, zum uns-übrig-Bleibenden. Daß für den Historismus Geschichte allein im Ergebnis zählt, schlägt zuletzt aber auf ihn selbst zurück, weil er so sein Wissen nicht legitimieren kann - außerhalb jedenfalls einer Methodologie, die - den Naturwissenschaften entlehnt - das Theoretische vom Gegenständlichen als das Formale vom Inhaltlichen zu trennen sucht. Denn die Verwechslung der Geschichte mit dem Gegebenen ist Bedingung einer aufs Methodische sich beschränkenden Selbstversicherung ${ }^{4}$. Daß die Kriterien wissenschaftlicher Rationalität selber historisch legitimiert werden müssen ${ }^{5}$, zeigt schließlich die Aporie der Selbstbegründung historistischen Denkens. Diese Grenzen methodischer Gewißheit liegen damit jeder Lektüre Schleiermachers voraus; sie kann sich nur-selber historistisch-darauf zurückfragen, indem sie das historistische Denken im hermeneutischen analysiert [Diese Überlegungen bleiben unausgeführt.].

Was die Lektüre Schleiermachers jenseits problem- oder begriffsgeschichtlicher Verobjektivierung aus solcher Selbstverständlichkeit des einordnenden Begreifens reißen kann, ist allein Erinnerung an Philosophie (s. 2.) und Geschichte (s. 3.) des hermeneutischen Denkens zu dessen Reflexion an ihm selbst. Denn der geisteswissenschaftliche Positivismus ist anders nicht in seiner beliebigen Selbstverlängerung zu unterbrechen, als indem ihm die Möglichkeit seines Anfangs gezeigt wird - dort, wo der Anspruch auf Wissenschaftlichkeit die geschichtliche Erfahrung zu objektivieren sucht. Philologie als gelehrte Praxis des 19. Jahrhunderts zu verstehen heißt dann, sie als gleichursprünglich mit den Kategorien ihres Denkens zu begreifen ${ }^{6}$. Dieser Aufweis mag an den frühen Schriften Schleiermachers möglich sein, soweit

45. J.Mittelstraß: Das Interesse der Philosophie an ihrer Geschichte, in: Studia philosophica 36, 1976.

5 S. H.Schnädelbach: Wissenschaftsgeschichte und Historismus, in: Studia Leibnitiana, Sonderheft 6, Wiesbaden 1977.

$6 \mathrm{Vgl}$. dazu R.Koselleck: "Erfahrungsraum" und "Erwartungshorizont" - zwei historische Kategorien, in: Soziale Bewegung und politische Verfassung (FS W.Conze), Stuttgart 1976. M.Foucault: Archäologie des Wissens (1969), Frankfurt/M. 1973. 
darin Kritik der vorgängigen rationalistischen Geschichtsauffassung deutlich, und damit dem historisch-hermeneutischen Denken ein philosophischer Bezug entdeckt wird.

\section{Lektüre}

Was Schleiermachers Religionsschrift zur Sprache bringt, ist nicht allein der theologische Gedanke, Religion sei Anschauung des Unendlichen, und auch nicht allein die historische These, die positiven Religionen seien Äußerungen dieser Anschauungen, vielmehr die Idee einer vermittelnden Einheit beider Sätze. Der Text ist selbst der Explikation dieser Idee gewidmet ${ }^{7}$ und damit Reflexion der aufklärerischen Religionskritik. Diese nämlich bewegte sich im Widerspruch von exklusiv moralisch definierter Religiosität einerseits und - in ideologiekritischer Absicht - gesellschaftlich definiertem religiösem Kultus andererseits. Das subjektive Wahre sprach ein gegen das öffentlich Geltende, an dem es sich beweisen wollte: der Konflikt von Moral und Politik markierte die Emanzipation des Bürgertums ${ }^{8}$, bis zur Vernunftvergötterung der französischen Revolutionäre. Diese Kritik am Staat und an der Kirche wurde notwendig historisch, sobald sie in der Ausübung von Macht deren Legitimation treffen wollte. Daher war die religionskritische Vernunft zur Interpretation von Offenbarung gezwungen ${ }^{9}$. Schleiermachers Text repetiert dieses seit Lessings Publikationen diskutierte Problem: er nimmt aber die Dialektik von Religion und Äußerung zurück ins geschichtliche Verhältnis von religiöser Anschauung und positiver Religion, indem er Ausgedrücktes mit den geschichtlichen Erscheinungen der Vergangenheit identifiziert. Es findet sich die traditionelle aufklärerische Anklage zerstört: die Entgegensetzung von guter und schlechter Praxis wird ihrer Voraussetzung überführt, Handeln psychologisch aus der Gesinnung zu verstehen und daran zu werten. Schleiermachers Text gibt jeden moralischen Apriorismus preis ${ }^{10}$. Das Problem guten Handelns in depravierter Kultur (worauf Kants geschichtsphilosophische Schriften rekurrieren) Iöst sich im Problem der Anschauung einer Äußerung als Äußerung einer Anschauung auf: die Reflexion der aufklärerischen Religionskritik behält deren Wertung nur als Urteil ohne 'psychologische' Begründung. Sie trägt sich als Forderung vor, die positiven Religionen religiös zu richten, d.h. anschauend ihre Individualität als Äußerung des "Geistes

$7 \mathrm{Vgl}$. H.Timm: Die heilige Revolution - Das religiöse Totalitätskonzept der Frühromantik, Frankfurt/M. 1978, S. $43 \mathrm{f}$.

$8 \mathrm{Vgl}$. R.Koselleck: Kritik und Krise (1959), Frankfurt/M. 1979.

9 S. G.E.Lessing: Über den Beweis des Geistes und der Kraft (1777), in: Werke Bd. VIII, München. S.a. L.Strauß: Die Religionskritik Spinozas als Grundlage seiner Bibelwissenschaft (1930), Darmstadt 1981.

$10 \mathrm{Vgl}$. W.Dilthey: Leben Schleiermachers, a.a.0., S. $114 \mathrm{ff}$. 
der Religion" $" 1$ zu verstehen. In diesem Anspruch ist offensichtlich der Gesichtspunkt der Geltung aufgegeben, der der Aufklärung noch im Gedanken religiöser Toleranz maßgeblich war, und die historische Faktizität aller Religionen eingeräumt. (Wirksam wurde dieser Gedanke vor allem für die Christologie: sobald nämlich Mythologie zur Wirklichkeit der Religionen gezählt wurde, durfte Jesus nicht mehr als vergötterter Mensch, sondern konnte als göttlicher Mensch begriffen werden ${ }^{12}$. Auch Wunder und Offenbarungen gehörten gleichsam zur Sprache der Religionen und nicht zur psychologisch erklärbaren Illusionswelt von Enthusiasmierten ${ }^{13}$,) Die Wirklichkeit der Religionen anzuerkennen, bedeutete somit, sie auf die Virtualität religiöser Anschauung zu beziehen: Jede positive Religion hat in dem, was religiöse Anschauung in ihr ausmacht, ihren Grund und umgekehrt: religiöse Anschauung bezieht sich auf positive Religionen als auf verwirklichte Anschauungen. So bringt die Religionsschrift Schleiermachers den Indifferenzpunkt von Geschichtsphilosophie und Hermeneutik zur Sprache: die historischen Individua aus dem Geist der Religion zu verstehen, heißt, sie darauf zurückzuführen - Anerkennung geschichtlicher Faktizität und Geschichte-Verstehen verweisen aufeinander. Die Analogie wird vom geschichtlich Vergangenen zum je Fremden gedacht. Damit gerät Religion als Geistiges, nicht als gesellschaftliches Phänomen in den Mittelpunkt der Reflexion, die daran ihre eigene Apologie formuliert, weil sie nicht mehr Kritik aus Selbstgewißheit üben kann: geschichtliche Individua verstehen, heißt den Geist finden, in dem das verstehende Subjekt selbst beschränkt sich fühlt: "Ich denke mich in tausend Bildungen hinein, um desto deutlicher die eigene zu erblicken." (Monologen) 14

Schleiermachers Reden über Religion stellen wie auch die Monologen im Gestus die Subjektivität der neuen Evidenz aus: auch darin bekundet sich Reflexion der traditionellen aufklärerischen Philosophie und Theologie. Für diese stand Selbstausdrücklichkeit im Dienst der allgemeinen Vernunft, die Kant radikal öffentlich nennt. Die Eigenheit jeder Rede gerierte sich als Anwaltschaft höherer Moralität, um nicht des Privaten verdächtigt zu werden. In dieser Haltung artikulierten Kirchenleute selbstverständlich Einspruch und

11 F.Schleiermacher: Über die Religion, a.a.0., S. 142.

12 F.Schleiermacher: Hermeneutik, a.a.0., S. 387 ff. Vgl, zum christologischen Geschichtsmodell K.Gründer: Erfahrung der Geschichte (1978), in: ders., Reflexion der Kontinuitäten, Göttingen 1982, S. 118 ff. Außerdem H.Renz: Geschichtsgedanke und Christusfrage. Zur Christusanschauung Kants und deren Fortbildung durch Hegel im Hinblick auf die allgemeine Funktion neuzeitlicher Theologie, Göttingen 1977.

$13 \mathrm{Vgl}$. in der Nachfolge Schleiermachers F.Chr.Baur: Symbolik und Mythologie oder die Naturreligion des Altertums, Stuttgart $1824 \mathrm{f}$.

14 F.Schleiermacher: Monologen (1800), Hamburg 1978 (Phil. Bibl. 84), S. 32 . 
Zuspruch auch bei politischem Anlaß, etwa der Französischen Revolution ${ }^{15}$. Die Predigt war in gängiger Übung dem historischen Wechselspiel des Menschlich-Allzumenschlichem gewidmet ${ }^{16}$. Ihr Gestus war die Anrede. Gleiches galt für die Schriften der biblischen Theologen, welche die Geistesgeschichte als Geschichte von Meinungen und Umständen, Ereignissen und Reaktionen dem Leser zur Entscheidung vorlegten ${ }^{17}$. Die wissenschaftliche Erkenntnis dessen, was gewesen ist, stand generell unter dem Vorbehalt moralischer Wertung, weswegen auch hier das rhetorische Engagement dem Ausgesagten gegenüber unwesentlich war: die Rede sollte - wie bei der Predigt - Mittel zum Zweck $\operatorname{sein}^{18}$. Die im aufklärenden Meinen vorausgesetzte Unabhängigkeit von Person und Sache, von aussagendem Subjekt und thematisiertem Gegenstand reflektieren Schleiermachers frühe Schriften auf das darin implizit versprochene Aufgehobensein in überindividueller Vernunft und setzen dieses als ausdrückliches Moment der Rede selbst. Der Mitteilungscharakter ist jeder Rede nicht kontingent, sondern ihr als Äußerung, als individuelles Allgemeines konstitutiv $^{19}$. So ist confessio, Monolog, symbolische Adresse der eigene Charakter religiöser Rede, Die Anerkennung der historischen Vielfalt der Religionen führt deshalb nicht zum praktischen Problem einer moralischen Wertung, sondern zur einfachen Frage der Verstehbarkeit eines Anderen. Es stellt sich das Problem des Verstehens von Individualitäten als Verstehen von eigenstrukturierten Äußerungen. Wie die Rede sich vom Urteil zur Selbstausdrücklichkeit einer Anschauung zurückzieht, so entwertet sich am Sachverhalt das Beurteilbare zugunsten des jeweils Eigenen, d.h. objektiv Fremden. Das Fremde ist Voraussetzung der eigenen Bildung: Es muß hervorgebracht werden, um erworben, angeeignet zu sein (Monologen) ${ }^{20}$. Die Positivität der Religionen verstehen heißt, ihnen als dem Anderen des Eigenen dessen Rede zu leihen. Der historische Gegenstand ist darum nicht, wie in der Aufklärung, Beispiel für das, was subjektive Vernunft einschränkt (und darum kri-

$15 \mathrm{Vgl}$. E.D.Junkin: Religion versus Revolution. The Interpretation of the French Revolution by German Protestant Churchmen 1789-1799, Austin (Texas) 1974 (Diss. Basel 1968).

16 Vgl. R.Krause: Die Predigt der späten deutschen Aufklärung (1770-1805), Stuttgart 1965.

$17 \mathrm{Vgl}$. beispielsweise in der biblischen Theologie F.V.Reinhard: Versuch über den Plan, welchen der Stifter der christlichen Religion zum Besten der Menschheit entwarf, 1789 (4. Aufl. 1798); J.J.Heß: Geschichte der drei letzten Lebensjahre Jesu, 3 Bde., Leipzig 1768-1772 (7. Aufl. 1823 ff.); J.S.Semler: Beantwortung der Fragmente eines Ungenannten, 1779; dazu vom Verf.: Zur "Konstruktion des Gegenstandes" in den Geisteswissenschaften - Eine historische Anmerkung, in: Ontologie und Wissenschaft, Philosophische und wissenschaftshistorische Untersuchung zur Frage der Objektkonstitution, hg. v. H.Poser u. H.W.Schütt, Berlin 1984.

$18 \mathrm{Vgl}$. J.S.Semler: Zur Revision der kirchlichen Hermeneutik und Dogmatik, Halle 1789; Vorbereitung zur theologischen Hermeneutik, 1761; Beantwortung ..., a.a.o. und andere Schriften.

19 F.Schleiermacher: Über die Religion, a.a.0., S. 101; vgl. dazu M.Frank: 
tisiert werden muß), sondern für das, was sie entgrenzt in das in ihr ursprünglich selbst eingebildete Andere hin ${ }^{21}$ (und darum als Ausdruck konstituiert werden muß).

Als Moment der in neuer Evidenz formulierten Geschichtslogik und verinnernden Subjektivität ist auch die hermeneutische Theorie Reflexion der rationalistischen Geschichtsauffassung, spezifisch der der Philologie. Diese überhob sich in der Erklärung von Dokumenten aus Motiven der Selbstrechtfertigung ihres eigenen Tuns: die Erklärungsmodelle sind verschiedenen Modifikationen eines Allgemeinbegriffs menschlicher Praxis nachgebildet ${ }^{22}$. (So wurden Leben und Lehre Jesu als Handeln aufgefaßt, was Untersuchungen über ZweckmäBigkeit, Absichten, Erfolg, Aufrichtigkeit etc. provozierte. So war auch die Analogie Jesus-Sokrates möglich.) Dieses Wirkungsverstehen wird im Ausdrucksverstehen reflektiert, indem der Widerspruch, ein Besonderes aus einem Allgemeinen zu erklären, ohne den Schritt vom Gegebenen zum Erklärenden zu begründen, aufgelöst wird, wenn beide Seiten im Verstehen als Akt, als innerem Handeln zusammengenommen werden ${ }^{23}$. Solchem von der Wut des Wissens befreiten Verstehen erscheint ein fremder Text nicht ausschließlich als Produkt einer Praxis, nicht als Dokument, vielmehr als geistiger Ausdruck, als zu lesender Text, damit als Quelle des eigenen Selbstverständnisses. (So erscheint hier die Analogie Jesus-Sokrates nur möglich im Problem der Schriftlichkeit, denn einzig die Texte der Apostel wie der Sokratiker sind Ausdruck einer geschichtlichen Wirksamkeit (ausgehend von Jesus, von Sokrates), nicht Dokument eines auch sonst erschließbaren Geschehens. ${ }^{24}$ )

Philologie führe gerade als kunstgemäßes Lesen zur Geschichtserkenntnis, formulieren Notizen Friedrich Schlegels von $1797^{25}$, die also behaupten, was das hermeneutische Denken zur Sprache bringt: Geschichte im Abstand zwischen Quelle und Interpretation zu begreifen. Der Anspruch des hermeneutischen Denkens, diesen totalen, tatsächlich jedoch relativen Abstand im Verstehen aufzulösen, behauptete negativ die Notwendigkeit, im Aneignen des Fremden die Fremdheit sich selbst gegenüber zu überwinden ${ }^{26}$. Das richtige Verstehen, dem die Regeln der Auslegungskunst gelten, hält die Schwebe zwischen Lesen (Referieren) und Schreiben (Reflektieren) ${ }^{27}$; die verstehende Das individuelle Allgemeine, Frankfurt/M., 1977.

20 S. F.Schleiermacher: Monologen, a.a.0., S. $77 \mathrm{ff}$.

$21 \mathrm{Vgl}$. ebenda, S. $68 \mathrm{ff}$.

22 S. E.Troeltsch: Religionswissenschaft und Theologie des 18. Jahrhunderts, in: Preußische Jahrbücher Bd. 114, 1903.

$23 \mathrm{Vgl}$. F.Schleiermacher: Monologen, a.a.O., S. 67 ff.; Hermeneutik, a.a.0., S. 384 .

$24 \mathrm{Vgl}$. F.Schleiermacher: Hermeneutik, a.a.0., S. 127.

25 Vgl. F.Schlegel: Philosophie der Philologie, hg. v. J.Körner, in: Logos XVII, 1908.

26 F.Schleiermacher: Monologen, a.a.0., S. 25 f.

27 Ders.: Hermeneutik, a.a.0., S. 75. 
Rede ist - paradox - sich soweit selbst gewiß, soweit sie sich mißtraut, Mißverstand überall annimmt. Darum besagt das Wort, die Rede eines Autors besser als er verstehen zu können, nicht Besserwissen durch Posterität oder emphatische Teilnahme; es drückt im Blick auf methodische Maximen die Unmöglichkeit unmittelbaren Verstehens, gerade Selbstverstehens, aus. Die Geschichtlichkeit alles Objektiven hat dessen Begriff in die Individualität gebannt, er ist nicht in der Vernunft zu finden, vielmehr im vermittelten Endlichen zu suchen: Das "Geschäft des Verstehens und Auslegens ... ist das allmähliche Sichselbstfinden des denkenden Geistes" (Hermeneutik) 28 .

Das hermeneutische Denken trauert über die Vergangenheit aller Geschichte: Verstehen zwingt, die Begriffe ins Spiel zu bringen; wem es für Erkenntnis gilt, muß sich als Einsatz wagen. Was aus frühen Texten Schellings und Schleiermachers mit gleicher Stimme spricht, ist das Bewußtsein der Verlorenen, der sündig Gefallenen, der Entfremdeten. Geschichtsphilosophie und Hermeneutik arbeiten ursprünglich am selben Problem der Selbstentäußerung, welche nicht durch Rückkehr zum Mythos, vielmehr durch reflektierte Ichentgrenzung, durch angestrengte (kunstgemäße) Anschauung begriffen werden soll. Medium ist daher die Erinnerung: Vergegenwärtigung als Innewerden erst gibt das Fremde als das Andere des Eigenen, enthüllt Geschichte um den Preis des Lebens im Augenblick. Das bürgerliche Denken der Epoche tauscht unerfüllte Gegenwart gegen erfüllte Vergangenheit und eine vage Zukunft, auf die es aus Erinnerung hofft ${ }^{29}$.

\section{Historismus}

Die vorstehende Skizze des hermeneutischen Denkens erweist dessen geschichtslogische Evidenz als Reflexion aufklärerischer Rationalität und Geschichtsauffassung. Der konstruierende Zugriff moralischer Subjektivität auf Geschichte als Gegebenes wird in der Konstitution einer Relation des Eigenen und des Fremden aufgehoben. An sich selbst ist damit die Form geisteswissenschaftlichen Arbeitens, d.h. der philologische Diskurs theoretisch ausgewiesen: Hermeneutisches Denken in actu zeigt sich nämlich nur in der Praxis der Interpretation. Unberührt von der philosophischen Verpflichtung auf ideales Verstehen besitzt es daher selbst eine Geschichte und kann in der ihm eigenen Anschauung alles Vergangenen als Faktum sich selbst gegenständlich werden. Diese Reflexion des Positivismus' hermeneutischen Denkens hat (nachträglich) den Titel Historismus erhalten.

Daß das Verstehen als interpretation Geschichte macht, findet sich in

28 Ebenda, S. 327 f.

29 S. I.Kant: Rezension von Herders Ideen zur Philosophie der Geschichte der Menscheit (1785), Akad. Ausg. Bd. VIII, S. 52; Mutmaßlicher Anfang der Menschengeschichte (1786), ebenda, S. 109. 
Texten Diltheys ausgedrückt, die auf die Praxis des gelehrten geisteswissenschaftlichen Diskurses gehen. Im Unterschied zur originären Reflexivität des hermeneutischen Denkens, die sich als Auflösung rationalistischer Subjektivität konstituiert und damit erst philologische Arbeit an Texten als Quellen provoziert, bildet die mit dem Titel Historismus belegte Reflexion wenn nicht als Ideologie ${ }^{30}$, so einfach als Eingeständnis der geisteswissenschaftlichen Gelehrsamkeit ${ }^{31}$ ein Moment dieser selbst. Die auch bei Dilthey unscharfe Identifikation von hermeneutischen und historischem Denken hat ihre Wahrheit gerade als innere Reflexion einer theoretischen Praxis, die den gängigen begrifflichen Unterscheidungen entgeht, wonach Geschichte-Verstehen entweder als Spezifikum allgemeiner Hermeneutik (Gadamer) ${ }^{32}$ oder als besondere Aufgabe der Historik (Droysen) ${ }^{33}$ exponiert wird. Universalhermeneutische und geschichtsphilosophische Bestimmungen des Historismus begreifen ihn dementsprechend nicht als Philosophie, sondern als das unglückliche Bewußtsein der Philologie. Sie verweisen so - obzwar im Bemühen kritischer überwindung - darauf, daß Historismus Titel für eine historische Erfahrung ist ${ }^{34}$, die vom wissenschaftlichen Positivismus nicht getrennt werden kann ${ }^{35}$. Sozusagen innerhalb des historistischen Denkens zu sortieren und etwas Positives, die Anerkennung universaler Geschichtlichkeit, von etwas Pejorativem, dem Positivismus der Forschung und dem Relativismus der Wertung, zu scheiden $^{36}$, bedeutet dann, in theoretischer Beflissenheit auseinanderzuhalten, was in der diskursiven Praxis zusammengehört.

Die historische Geschichtsauffassung in den Geisteswissenschaften dokumentiert sich vor allem im Epochendenken: daß Zeitalter wohl, nicht aber Fortschritt aus der Geschichte gelesen werden können, folgt logisch der Ansicht, "daß der objektive Geist eine gegliederte Ordnung in sich enthält" 37 . Verstehen als Vergegenwärtigung im Rahmen dieser Ordnung erhält den Charakter der Restitution von Ursprünglichkeit, also den Schein verlustlosen Innewerdens. Dabei wird die Vermitteltheit, die Bildlichkeit des Verstehens - Form seiner Erkenntnis - material in die Textualität des geschichtlich Positiven (beispielsweise Religion) projiziert, worin das Allgemeine (der Geist)

30 F.Meinecke: Die Entstehung des Historismus, Berlin 1936.

31 K.Mannheim: Historismus, in: Archiv für Sozialwissenschaft und Sozialgeschichte 52, 1924. E.Troeltsch: Der Historismus und seine Überwindung, Berlin 1924. W.Dilthey: Die Typen der Weltanschauung und ihre Ausbildung in den metaphysischen Systemen (1911), in: Ges.Schriften VIII.

32 S. H.G.Gadamer: Wahrheit und Methode, Tübingen $1965^{2}$.

33 S. H.Schnädelbach: Geschichtsphilosophie nach Hegel. Die Probleme des Historismus, Freiburg 1974.

34 S. K,Löwith: Die Dynamik der Geschichte und der Historismus, 1952.

35 5. K.Heussi: Die Krisis des Historismus, Tübingen 1932.

36 Vgl. H.Schnädelbach: Geschichtsphilosophie ... a.a.0. (Anm. 39).

37 W.Dilthey: Der Aufbau der geschichtlichen Welt in den Geisteswissenschaften, in: Ges. Schriften Bd. VII, S. 208 ; vgl. a. S. $311 \mathrm{f}, 357 \mathrm{f}$, 
lesbar scheint. Das Bewußtsein der Epochenstruktur von Geschichte entlastet so von der eigentlichen hermeneutischen Anstrengung einer Versicherung dieses Allgemeinen. Der unbestimmte Trost, es in der Betrachtung des Vergangenen immer schon zu besitzen, ist nur Kompensation der Anerkennung sinnloser Faktizität an sich selbst. Damit wird gerade die Form hermeneutischen Denkens, die geistige Vermittlung des Eigenen mit dem Fremden nicht im Moment der Bewegung, sondern im Ergebnis begriffen: Sobald die philologische Arbeit die Objektivität der Geschichte in den Produkten ihrer Auslegung anschaut, reflektiert sie sich instrumental als aneignende Methode. Fortschritt gibt es dann allein als gesteigerten "Erwerb geistiger Dinge" ${ }^{38}$, als materiale Bereicherung des Erinnerns, wie sie sich im vermehrten Textkorpus der Gelehrsamkeit dokumentiert. Historistisch ist das hermeneutische Denken also als philologische Praxis, weil schriftlich die Dialektik von Teil und Ganzem aus gesprächshafter Flüssigkeit im Text erstarrt. Die Unvermeidlichkeit der philologischen Praxis, als Praxis Folgen zu zeitigen, etwas zu ergeben, entfremdet das hermeneutische Denken im Ergebnis selbst - was der theoretische Einspruch philosophischer Hermeneutik gegen den Historismus (Gadamer) nicht einmal berührt ${ }^{39}$. Auch jede gegenwärtige Lektüre Schleiermacherscher Texte wird sich nicht verstehend fragloser Annäherung widmen können, solange ihr spezifisches Vorurteil als Unternehmung im philologischen Diskurs geltend bleibt: sie kann sich nur inner-historistisch auf den Anfang des hermeneutischen Denkens zurückbeziehen, dem sie selbst angehört.

38 Ebenda, S. 215.

$39 \mathrm{Vgl}$. H.G.Gadamer: Wahrheit ..., a.a.0. (Anm, 38). 